\title{
Domain-specific characterisation of early cognitive impairment following spontaneous intracerebral haemorrhage
}

Gargi Banerjee ${ }^{1 *}$, Mary Summers ${ }^{2 *}$, Edgar Chan ${ }^{1,2}$, Duncan Wilson ${ }^{1}$, Andreas Charidimou ${ }^{3}$, Lisa Cipolotti $^{2,4}$, David J Werring ${ }^{1}$

* These authors contributed equally

${ }^{1}$ Stroke Research Centre, Department of Brain Repair and Rehabilitation, UCL Institute of Neurology and the National Hospital for Neurology and Neurosurgery, London, UK

${ }^{2}$ Department of Neuropsychology, National Hospital for Neurology and Neurosurgery, Queen Square, London, UK

${ }^{3}$ Hemorrhagic Stroke Research Group, Massachusetts General Hospital and Harvard Medical School, Boston, Massachusetts, USA

${ }^{4}$ Dipartimento di Scienze Psicologiche, Pedagogiche e della Formazione, Università degli Studi di Palermo, Palermo, Italy.

\section{Corresponding author:}

Professor David J Werring

UCL Stroke Research Centre, Department of Brain Repair and Rehabilitation, UCL Institute of Neurology, Russell Square House, 10 - 12 Russell Square, London WC1B 5EH, UK.

Tel: +44 (0)20 3108 7493; Fax: +44 (0)20 7833 8613; Email: d.werring@ucl.ac.uk

\section{Key words:}

Cerebral amyloid angiopathy; small vessel disease; intracerebral haemorrhage; cognitive impairment; stroke 


\section{ABSTRACT}

Cognitive deficits after spontaneous intracerebral haemorrhage (ICH) are common and result in functional impairment, but few studies have examined deficits across cognitive domains in the subacute phase. This study aims to describe the cognitive profile following acute ICH and explore how cerebral amyloid angiopathy (CAA) may impact performance. We retrospectively reviewed 187 consecutive patients with ICH (mean age 58.9 years, 55.6\% male) with available imaging and neuropsychological data (median 12 days after stroke). In our cohort, $84 \%(\mathrm{n}=158)$ were impaired in at least one cognitive domain and 65\% $(\mathrm{n}=122)$ in two or more domains. Deficits in non-verbal IQ (76.6\%), information processing speed (62.4\%) and executive functions (58.1\%) were most common. Patients with lobar ICH $(n=92)$ had more deficits in naming and visual perception than those with non-lobar ICH, but not in adjusted analyses. Patients with probable CAA $(n=21)$ had more deficits in verbal IQ, visual perception and executive functions than those without probable CAA; in adjusted analyses, probable CAA predicted impairment in verbal IQ (OR 38.6, 95\% CI 3.2 to $465.4, \mathrm{p}=0.004$ ) and executive function (OR 3.4, 95\% CI 1.0 to $11.7, \mathrm{p}=0.050$ ). We conclude that cognitive deficits following ICH are common across domains, and that those with CAA appear to have a different cognitive profile. Replication of this work in larger cohorts will be important for confirming and further quantifying these observations. 


\section{INTRODUCTION}

Cognitive deficits that occur as a consequence of spontaneous (non-traumatic) intracerebral haemorrhage $(\mathrm{ICH})$ are common and can result in significant functional impairment[1]. The association between ICH and the development of subsequent dementia is increasingly recognised, with more than a quarter of patients who were dementia-free at the time of their ICH developing dementia within 4 years[2]. Moreover, there is increasing evidence that the presence of structural imaging markers of cerebral small vessel disease (SVD) at the time of ICH might predict subsequent cognitive decline[2,3]; this is of interest as the two commonest SVD, cerebral amyloid angiopathy (CAA) and deep perforating arteriopathy, are associated both with cognitive impairment and ICH[4, 5]. In particular, the presence of structural imaging markers of CAA appears to be associated with subsequent progression to dementia[2]. Additionally, the presence of cognitive impairment prior to ICH is common (estimated at 16.7\%), suggesting that the development of cognitive deficits after ICH is likely due to a combination of damage due to the ICH itself together with more longstanding impairment due to the presence of underlying SVD[1, 5, 6].

Whilst the association between ICH and cognitive impairment is recognised, less is known about the extent and nature of these deficits. Most studies investigating cognitive deficits following ICH have focused on the presence or absence of dementia, using global measures of cognition such as the MiniMental State Examination (MMSE) or the Montreal Cognitive Assessment (MoCA)[2, 7]. More recent studies have examined domain-specific impairment following $\mathrm{ICH}$, but investigations have been limited to a select number of domains; mainly executive functions, verbal memory and information processing speed[8-10]. The prevalence of impairments in other domains such as nonverbal memory, visuo-perceptual functions and naming, in addition to changes in general intellectual functioning after ICH remain largely unknown, yet these domains are frequently affected early following ischaemic strokes, and have important predictive validity for functional outcomes[11].

This study therefore aims to describe the cognitive profile of patients presenting with ICH during the early (subacute) phase, a time for which there is limited data currently available but clinical 
assessments frequently take place. We compare the cognitive domains affected in lobar vs. non-lobar haemorrhage and those with vs. without probable CAA to further investigate the potential role of SVD pathologies in cognition.

\section{MATERIALS AND METHODS}

\subsection{Subjects}

Data from 187 consecutive patients treated for stroke at the National Hospital for Neurology and Neurosurgery (NHNN), between May 2009 and May 2015, were retrospectively screened for inclusion into the study. All patients had to have a spontaneous ICH confirmed by neuroimaging (either CT or MRI) for which there was no obvious secondary cause and available neuropsychological data. Demographic and clinical information collected comprised of age, sex, years of education, lesion laterality and the time between stroke onset and neuropsychological assessment. Data on pre-existing comorbidities and cognitive impairment prior to stroke was not available.

This study was conducted in accordance with a Service Evaluation Agreement approved by the local Research Ethics Committee (joint UCL Institute of Neurology / National Hospital for Neurology and Neurosurgery); this agreement provides permission to review anonymised data from patients presenting to our stroke service with intracranial haemorrhage, and waives the need for written consent.

\subsection{Neuropsychological measures}

All patients were assessed using a tailored neuropsychological assessment by a clinical neuropsychologist, blind to the aims of the current study, as a part of standard routine care. The comprehensive neuropsychological assessment evaluated seven cognitive domains: premorbid intellectual functioning, current intellectual functioning, verbal and visual memory, naming, visuospatial perception, information processing speed and executive function (detailed within the Online Supplement). As this was a retrospective study, patients were administered a subset of tests from each 
domain deemed suitable by the clinical neuropsychologist at the time of testing. Performance was scored according to standard clinical procedure and published normative data. Performance at or below the 5th percentile on any one test were taken to indicate impairment in that domain. For the executive domain, failure on two or more standardized tests was taken to indicate impairment except when screening measures were administered, whereby failure on only one test was taken to indicate impairment. For intellectual functioning, impairment was classified as a difference of at least 10 points or more between either the Verbal or Performance IQ measures and the respective premorbid functioning score on the National Adult Reading Test. Further details regarding the classification procedure have been previously described[12]. In addition to the cognitive domains, symptoms of anxiety and depression were also assessed and clinical significance was classified according to published cut-offs. For patients experiencing delirium or acute confusional states, the neuropsychological assessment was performed at a timepoint when patients was no longer delirious (based on an evaluation by the testing neuropsychologist). Tests were selected to minimize the effects of dominant hand weakness.

\subsection{Imaging acquisition and analysis}

All imaging analysis was performed by a single trained rater (DW). Scans were reviewed and patients were initially classified as having either lobar or non-lobar bleeds and then having either probable or non-probable CAA based on the original Boston criteria. ICH location was classified as non-lobar if the haemorrhage localised to one of the following regions: cerebellum, brainstem, thalamus, basal ganglia, caudate, deep white matter and corpus callosum. Haemorrhages in the frontal, temporal, occipital and parietal lobes without extension into the aforementioned deep regions were considered as lobar. Probable CAA was defined using the original Boston criteria[13]; the criteria was met if 2 lobar ICH were present on CT, or if a single lobar ICH was present in addition to lobar cerebral microbleeds on MRI with no further macro- or microhaemorrhages in the deep structures listed above. Cerebral microbleeds were identified in accordance with consensus guidelines[14].

\subsection{Statistical Analysis}


Statistical analyses were performed using Stata (Version 11.2). Baseline characteristics were compared using Chi-squared tests for categorical variables, independent t-tests for normally distributed continuous variables and Mann-Whitney $U$ tests for continuous variables that were not normally distributed. We conducted two separate comparisons to investigate whether there were any differences in the prevalence of domain-specific cognitive impairment between the predefined clinical subgroups: lobar vs. non-lobar, and probable CAA vs. non-probable CAA. Adjusted logistic regression was then performed for neuropsychological domains of interest $(\mathrm{p}<0.10)$ identified during the univariate comparisons; adjustments were made for demographic variables that were different $(\mathrm{p}<0.10)$ during baseline comparisons.

\section{RESULTS}

In this cohort ( $\mathrm{n}=187$; median time to neuropsychological assessment 12 days), 49\% ( $\mathrm{n}=92)$ had lobar haemorrhages and $11 \%(\mathrm{n}=21)$ met diagnostic criteria for probable CAA; 75\% had brain MRI available ( $n=140)$. The demographic and clinical characteristics of the patient sample are described in Tables 1 and 2. 


\begin{tabular}{|c|c|c|c|c|}
\hline & Total & Lobar ICH & Non-lobar ICH & p value \\
\hline $\mathrm{n}$ & 187 & 92 & 95 & - \\
\hline Age in years, mean $(\mathrm{SD})$ & $58.9(15.9)$ & $61.6(16.4)$ & $56.3(15.0)$ & 0.022 \\
\hline Sex, male $(\%)$ & $104(55.6)$ & $44(47.8)$ & $60(63.2)$ & 0.035 \\
\hline Years of education, median (IQR) & $13.0(6.0)$ & $11.0(6.0)$ & $13.0(5.0)$ & 0.555 \\
\hline \multicolumn{5}{|l|}{ Side of ICH, number (\%) } \\
\hline Left & $74(39.6)$ & $34(37.0)$ & $40(42.1)$ & \multirow{3}{*}{0.211} \\
\hline Right & $107(57.2)$ & $53(57.6)$ & $54(56.8)$ & \\
\hline Bilateral & $6(3.2)$ & $5(5.4)$ & $1(1.1)$ & \\
\hline Median days between stroke and assessment, days, median (IQR) & $12.0(30.0)$ & $13.0(27.5)$ & $10.0(31.0)$ & 0.245 \\
\hline \multicolumn{5}{|l|}{ Cognitive Domains } \\
\hline \multicolumn{5}{|l|}{ Intellectual functioning } \\
\hline Verbal IQ (n=55), number impaired (\%) & $25(45.5)$ & $17(50.0)$ & $8(38.1)$ & 0.389 \\
\hline Non-verbal IQ ( $\mathrm{n}=47)$, number impaired (\%) & $36(76.6)$ & $23(82.1)$ & $13(68.4)$ & 0.276 \\
\hline \multicolumn{5}{|l|}{ Memory } \\
\hline Verbal memory $(\mathrm{n}=125)$, number impaired $(\%)$ & $35(28.0)$ & $21(32.8)$ & $14(23.0)$ & 0.220 \\
\hline Non-verbal memory $(\mathrm{n}=134)$, number impaired $(\%)$ & $57(42.5)$ & $28(41.8)$ & $29(43.3)$ & 0.861 \\
\hline Naming $(\mathrm{n}=164)$, number impaired $(\%)$ & $61(37.2)$ & 37 (44.6) & $24(29.6)$ & 0.048 \\
\hline \multicolumn{5}{|l|}{ Visuo-perception } \\
\hline Visuo-perceptual $(\mathrm{n}=164)$, number impaired $(\%)$ & $40(24.4)$ & $25(31.3)$ & $15(17.9)$ & 0.046 \\
\hline Visuo-spatial $(\mathrm{n}=123)$, number impaired $(\%)$ & $36(29.3)$ & $21(35.6)$ & $15(23.4)$ & 0.139 \\
\hline Executive functions $(\mathrm{n}=172)$, number impaired (\%) & $100(58.1)$ & $46(54.1)$ & $54(62.1)$ & 0.291 \\
\hline Speed of processing $(\mathrm{n}=117)$, number impaired $(\%)$ & $73(62.4)$ & $40(67.8)$ & $33(56.9)$ & 0.224 \\
\hline \multicolumn{5}{|l|}{ Mood } \\
\hline Anxiety $(\mathrm{n}=81)$, number impaired (\%) & $39(48.1)$ & $18(52.9)$ & $21(44.7)$ & 0.463 \\
\hline Depression $(\mathrm{n}=91)$, number impaired $(\%)$ & $47(51.7)$ & $17(47.2)$ & $30(54.6)$ & 0.494 \\
\hline
\end{tabular}

Table 1: Demographic, clinical and cognitive domain-specific comparisons between those with and without lobar ICH 
Within the whole sample, $84 \%(\mathrm{n}=158)$ were impaired in at least one cognitive domain and $65 \%$ $(\mathrm{n}=122)$ in two or more domains. The most common impairment was decline in non-verbal IQ (76.6\%), followed by impairment in information processing speed and executive functions $(62.4 \%$ and $58.1 \%$ respectively). High proportions of patients were also found to have a decline in verbal IQ and non-verbal memory ( $45.5 \%$ and $42.5 \%$ respectively) whereas impairment in naming, visuoperceptual and visuo-spatial processing and verbal memory was less common. Around half of the total sample presented with anxiety and depression symptoms in the clinically abnormal range.

\subsection{Comparison between patients with lobar versus non-lobar ICH}

In our cohort, patients with lobar ICH were older (mean age 61.6 vs 56.3 years, $\mathrm{p}=0.022$ ) and less likely to be male $(44 \%$ vs $60 \%, \mathrm{p}=0.035)$. There were no differences in educational years, stroke lateralisation or time between stroke and assessment.

More patients with lobar ICH had impairments in two or more cognitive domains compared with nonlobar ICH (73\% vs 59\%, p=0.064). Comparisons between the separate cognitive domains are shown in Table 1. There were differences between those with and without lobar haemorrhage in naming (44.6\% vs $29.6 \%$ impaired, $\mathrm{p}=0.048)$ and visual perception ( $31.3 \%$ vs $17.9 \%$ impaired, $\mathrm{p}=0.046)$. In analyses adjusted for age and sex, the associations with deficits in naming (OR 1.8, 95\% CI 0.9 to 3.5 , $\mathrm{p}=0.080)$ and visual perception $(\mathrm{OR} 1.9,95 \%$ CI 0.88 to $4.0, \mathrm{p}=0.106)$ were no longer evident (Figure 1A). 
A

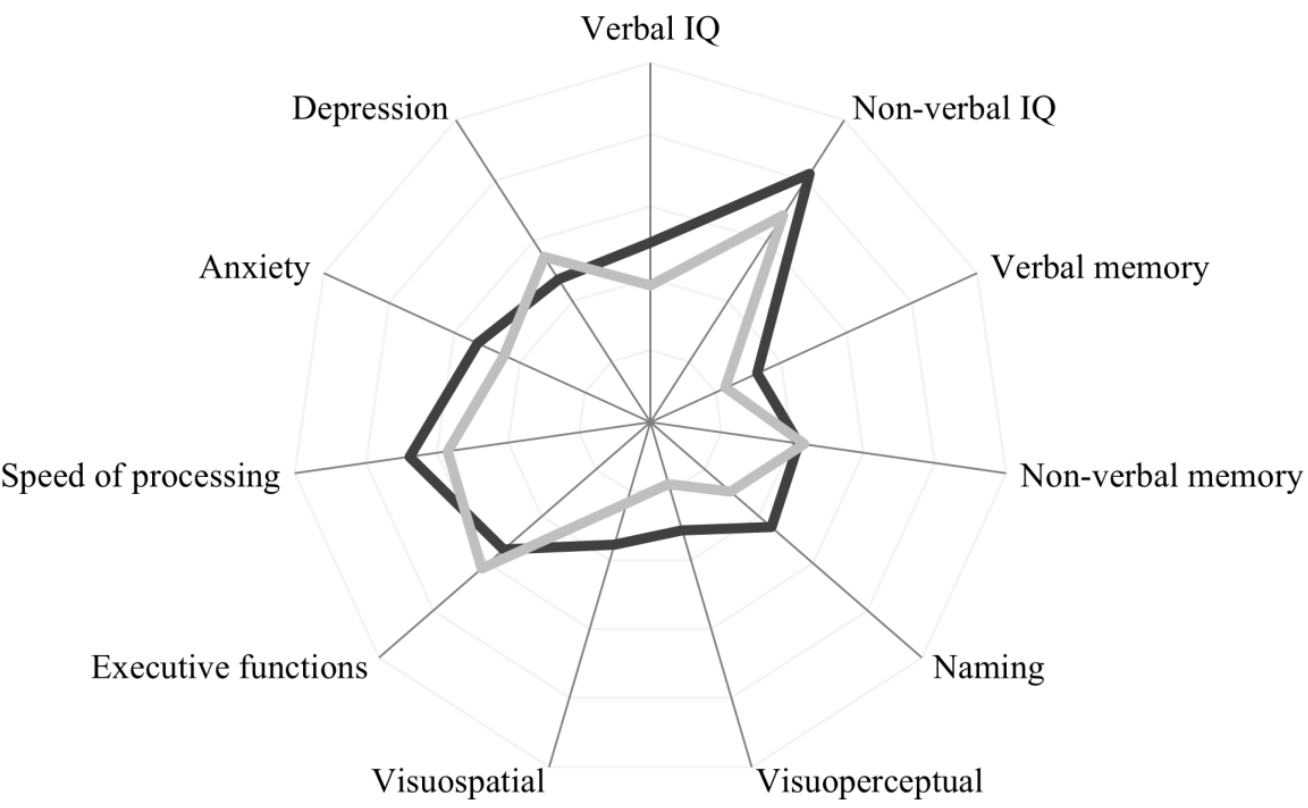

$\longrightarrow$ Lobar ICH Non-lobar ICH

B

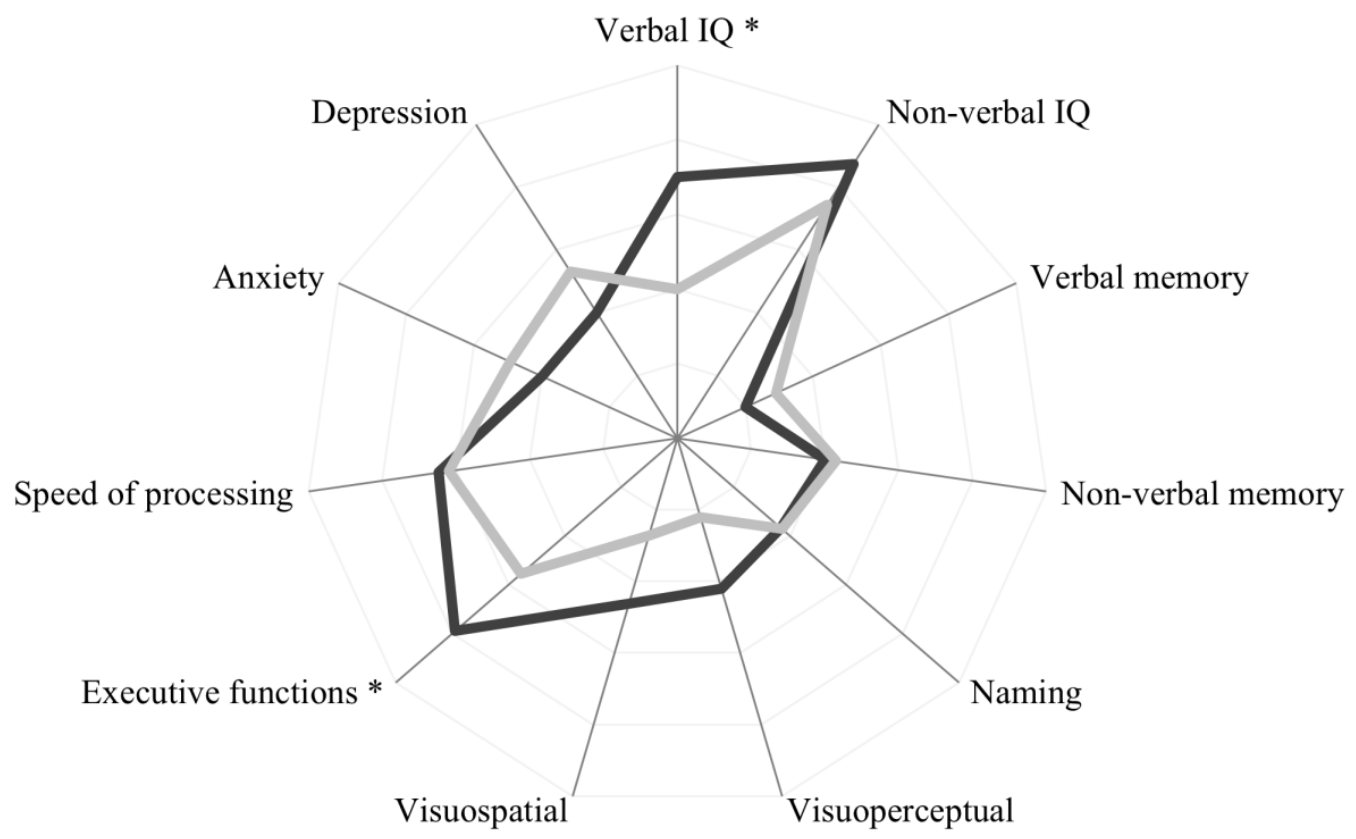

$\longrightarrow$ Probable CAA Non-probable CAA

Figure 1: Comparison of domain-specific impairments following ICH.

Concentric lines represent increasing degrees of impairment in $20 \%$ increments $(0 \%$ in the centre; $100 \%$ for outer ring).

Panel A: Comparison of lobar vs non-lobar ICH. No significant associations in adjusted logistic regression (adjusted for age and sex).

Panel B: Comparison of probable CAA vs non-probable CAA.

$* \mathrm{p}<0.05$ in adjusted logistic regression (adjusted for age and haemorrhage location). 


\subsection{Comparison between patients with and without probable CAA}

Patients with probable CAA were older (mean age 73.5 vs 57.1 years, $\mathrm{p}<0.00001$ ) and more likely to have bilateral haemorrhages $(\mathrm{n}=3,14.3 \%$, vs $\mathrm{n}=3,1.8 \%, \mathrm{p}=0.009)$. There were no differences in sex, educational years or time between stroke and assessment.

More patients with probable CAA had impairments in two or more cognitive domains ( $84 \%$ vs $64 \%$, $\mathrm{p}=0.149$ ) than non-CAA patients. Comparisons between probable CAA and non-CAA for the separate cognitive domains are shown in Table 2. There were differences between those with and without probable CAA in verbal IQ (70.0\% vs $40.0 \%$ impaired, $\mathrm{p}=0.085)$, visual perception $(42.1 \%$ vs $22.1 \%$ impaired, $\mathrm{p}=0.056)$ and executive functions $(79.0 \%$ vs $55.6 \%$ impaired, $\mathrm{p}=0.051)$. In adjusted analyses (adjusting for age and lesion side; Figure 1B), a probable CAA diagnosis was a predictor of impairment in verbal IQ (OR 38.6, 95\% CI 3.2 to $465.4, \mathrm{p}=0.004)$ and executive function (OR 3.4, 95\% CI 1.0 to $11.7, \mathrm{p}=0.050)$, but not visual perception (OR 1.9, 95\% CI 0.67 to $5.68, \mathrm{p}=0.223$ ). 


\begin{tabular}{|c|c|c|c|c|}
\hline & Total & Probable CAA & Non-probable CAA & p value \\
\hline $\mathrm{n}$ & 187 & 21 & 166 & - \\
\hline Age in years, mean $(\mathrm{SD})$ & $58.9(15.9)$ & $73.5(10.3)$ & $57.1(15.5)$ & $<0.00001$ \\
\hline Sex, male (\%) & $104(55.6)$ & $9(42.9)$ & $95(57.2)$ & 0.212 \\
\hline Years of education, median (IQR) & $13.0(6.0)$ & $13.5(5.5)$ & $13.0(6.0)$ & 0.513 \\
\hline \multicolumn{5}{|l|}{ Side of ICH, number $(\%)$} \\
\hline Left & 74 (39.6) & $8(38.1)$ & $66(39.8)$ & \multirow{3}{*}{0.009} \\
\hline Right & $107(57.2)$ & $10(47.6)$ & $97(58.4)$ & \\
\hline Bilateral & $6(3.2)$ & $3(14.3)$ & $3(1.8)$ & \\
\hline Median days between stroke and assessment, days, median (IQR) & $12.0(30.0)$ & $13.0(5.0)$ & $12.0(32.0)$ & 0.515 \\
\hline \multicolumn{5}{|l|}{ Cognitive Domains } \\
\hline \multicolumn{5}{|l|}{ Intellectual functioning } \\
\hline Verbal IQ (n=55), number impaired (\%) & $25(45.5)$ & $7(70.0)$ & $18(40.0)$ & 0.085 \\
\hline Non-verbal IQ $(n=47)$, number impaired $(\%)$ & $36(76.6)$ & $7(87.5)$ & $29(74.4)$ & 0.424 \\
\hline \multicolumn{5}{|l|}{ Memory } \\
\hline Verbal memory $(\mathrm{n}=125)$, number impaired $(\%)$ & $35(28.0)$ & $3(20.0)$ & $32(29.1)$ & 0.462 \\
\hline Non-verbal memory $(\mathrm{n}=134)$, number impaired $(\%)$ & $57(42.5)$ & $6(40.0)$ & $51(42.9)$ & 0.833 \\
\hline Naming $(\mathrm{n}=164)$, number impaired $(\%)$ & $61(37.2)$ & $7(36.8)$ & $54(37.2)$ & 0.973 \\
\hline \multicolumn{5}{|l|}{ Visuo-perception } \\
\hline Visuo-perceptual $(\mathrm{n}=164)$, number impaired $(\%)$ & $40(24.4)$ & $8(42.1)$ & $32(22.1)$ & 0.056 \\
\hline Visuo-spatial $(\mathrm{n}=123)$, number impaired $(\%)$ & $36(29.3)$ & $6(46.2)$ & $30(27.3)$ & 0.157 \\
\hline Executive functions ( $\mathrm{n}=172)$, number impaired (\%) & $100(58.1)$ & $15(79.0)$ & $85(55.6)$ & 0.051 \\
\hline Speed of processing $(n=117)$, number impaired $(\%)$ & $73(62.4)$ & $11(64.7)$ & $62(62.0)$ & 0.831 \\
\hline \multicolumn{5}{|l|}{ Mood } \\
\hline Anxiety $(\mathrm{n}=81)$, number impaired (\%) & $39(48.1)$ & $4(40.0)$ & $35(49.3)$ & 0.582 \\
\hline Depression ( $\mathrm{n}=91)$, number impaired (\%) & $47(51.7)$ & $4(40.0)$ & $43(53.1)$ & 0.435 \\
\hline
\end{tabular}

Table 2: Demographic, clinical and cognitive domain-specific comparisons between those with and without probable CAA 


\section{DISCUSSION}

Our study provides detailed description of the cognitive profile of patients during the early (subacute) phase following ICH (median 12 days after stroke). We found that a large proportion of patients (84\%) were impaired in one or more cognitive domains, with $65 \%$ impaired in two or more domains. Similar to previous findings, impairments in information processing speed and executive functions were common. However, our comprehensive assessment revealed that deficits in non-verbal IQ were most common, a domain that has not been investigated in previous studies. Similarly, deficits in other domains not routinely considered such as non-verbal memory, visuo-perceptual functions and naming were also evident. Patients with lobar ICH and those with probable CAA had higher rates of overall impairment. Although we identified deficits in naming and visual perception in those with lobar ICH compared to those without, these associations were not evident in adjusted analyses. By contrast, in adjusted analyses, those with probable CAA were significantly more likely to have deficits in verbal IQ and executive functions compared with those without probable CAA. Together, these findings suggest that the underlying SVD and not just ICH location may influence cognitive performance in the subacute phase following ICH.

Whilst the frequency of impairment that we report is similar to another acutely assessed acute stroke sample (82.4\%)[15], we did not find statistically significant differences between those with lobar and non-lobar ICH in specific cognitive domains. This is in contrast with previous studies which demonstrated an association between lobar ICH and cognitive impairment during early (within 3 weeks; mean 7.9 days)[15] and longer term (median 3.8 years)[7] follow up. This discrepancy may reflect that these previous studies used global measures which are more likely to capture impairments across multiple domains; this is consistent with our data, which did show that those with lobar ICH are more likely to have impairments in two or more domains than those with non-lobar ICH. However, when each domain is considered individually, the frequency of impairment does not differ between the lobar and non-lobar groups, which may suggest that lobar ICH results in a greater overall burden of impairment rather than a unique cognitive profile. 
Whilst we did not identify differences between those with lobar and non-lobar ICH in our cohort, there were differences between those with probable CAA and those without. This supports the suggestion that cognitive impairment in CAA is not only due to the disruption of eloquent cortical areas by direct lobar ICH damage, but also because of more widespread underlying CAA-related SVD damage. Structural markers of SVD are associated with disruptions of network efficiency[16, 17], and it has been hypothesised that SVD damage may specifically impact tasks that require integration of different brain areas, as these are particularly susceptible to "disconnection"[18]. The concept of acute or early cognitive impairment following ICH (in contrast to late or delayed onset) is recognised[1, 6], and thought to be due to direct consequences of the haemorrhage (for example, haematoma size and location), whereas delayed or late impairment, is thought to be driven predominantly by the small vessel processes[1]; this is supported by evidence that the presence of imaging markers of CAA at the time of ICH increases the risk of subsequent dementia[2]. Our results suggest that CAA (and thus potentially other small vessel pathologies) may also have an important role in cognitive impairment more acutely following ICH.

Patients with probable CAA were significantly more likely to have impairment in verbal IQ and executive functions compared with patients without probable CAA. Our results complement previous findings that show CAA is associated with increased executive dysfunction[8,9], but also suggest that CAA is also associated with decreased verbal IQ. Taken together with the finding that non-verbal IQ was the most commonly affected domain within the whole cohort, the increased frequency of verbal IQ impairment most likely reflects a more pronounced global general intellectual under-functioning in CAA. Following from this, the increased frequency of executive dysfunction in probable CAA might be a consequence of this global under-functioning, given that executive functions are thought to be sensitive to non-specific brain compromise[19]. The relationship between intellectual functioning and executive dysfunction in CAA warrants further study.

The main strengths of our study are that we included consecutive patients presenting with ICH to our stroke service, all with detailed neuropsychology testing performed. However, there are also some 
limitations. Having examined a range of cognitive domains, there is a potential issue of multiple comparisons. We chose to adopt an exploratory "hypothesis-generating" approach, and thus have not used a Bonferroni correction, which in this context would be overly-stringent adjustment given the inter-correlation of different cognitive scores within individuals. Some patients were unable to complete all aspects of the neuropsychological battery, resulting in small sample sizes for some domains. Patients unable to perform any aspects of the battery were not included in our study, so there is likely to be some underestimation of the true severity of impairment post-ICH. Not all patients with ICH were referred for neuropsychological testing, which could lead to both underestimation (due to exclusion of those felt to be too impaired for referral) and overestimation (due to exclusion of those felt to be too high-functioning for referral) of the deficits that we describe. CAA was diagnosed using $\mathrm{CT}$ in some cases, raising the possibility that some cases of CAA were missed or misclassified given the low diagnostic specificity of the Boston criteria based only on brain CT scans. We did not have information about previous ICH and so did not adjust our analyses for this; again, this could potentially confound the CAA group results. Additionally, we did not have any measures of prehaemorrhage cognitive function, and, given that the presence of cognitive impairment prior to ICH is common $[1,5]$, it is not possible to distinguish between those deficits present prior to ICH from those occurring subsequently. Moreover, we did not have longitudinal data for our patients, and so are unable to comment on whether the deficits observed persist beyond the early post-ICH phase.

\section{CONCLUSIONS}

We report that cognitive deficits acutely following ICH are common, and that those with CAA appear to have a distinct cognitive profile, adding to evidence that CAA has an independent effect on cognition, not just related to lobar ICH location, and that this effect may influence cognitive performance in the early period following acute ICH. Ongoing work aimed at identifying the most prevalent deficits following ICH (classified by underlying SVD as well as location) will be essential for allowing tailored rehabilitation approaches to maximize functional gains. 


\section{FUNDING}

GB receives funding from the Rosetrees Trust. DJW receives research support from the Stroke Association, the British Heart Foundation and the Rosetrees Trust. This work was undertaken at UCLH/UCL which receives a proportion of funding from the Department of Health's National Institute for Health Research (NIHR) Biomedical Research Centres funding scheme.

\section{CONFLICTS OF INTEREST/DISCLOSURES}

The authors report no disclosures or conflicts of interest relevant to the manuscript. 


\section{REFERENCES}

[1] L. Xiong, Y.D. Reijmer, A. Charidimou, C. Cordonnier, A. Viswanathan, Intracerebral hemorrhage and cognitive impairment, Biochimica et biophysica acta 1862(5) (2016) 939-44.

[2] S. Moulin, J. Labreuche, S. Bombois, C. Rossi, G. Boulouis, H. Henon, A. Duhamel, D. Leys, C. Cordonnier, Dementia risk after spontaneous intracerebral haemorrhage: a prospective cohort study, The Lancet. Neurology 15(8) (2016) 820-829.

[3] M.R. Benedictus, A. Hochart, C. Rossi, G. Boulouis, H. Henon, W.M. van der Flier, C. Cordonnier, Prognostic Factors for Cognitive Decline After Intracerebral Hemorrhage, Stroke; a journal of cerebral circulation 46(10) (2015) 2773-8.

[4] G. Banerjee, D. Wilson, H.R. Jager, D.J. Werring, Novel imaging techniques in cerebral small vessel diseases and vascular cognitive impairment, Biochimica et biophysica acta 1862(5) (2016) 92638.

[5] G. Banerjee, D. Wilson, G. Ambler, K. Osei-Bonsu Appiah, C. Shakeshaft, S. Lunawat, H. Cohen, T.D. Yousry, G.Y.H. Lip, K.W. Muir, M.M. Brown, R. Al-Shahi Salman, H.R. Jager, D.J. Werring, C.-. Collaborators, Cognitive Impairment Before Intracerebral Hemorrhage Is Associated With Cerebral Amyloid Angiopathy, Stroke; a journal of cerebral circulation 49(1) (2018) 40-45. [6] A. Biffi, D. Bailey, C.D. Anderson, A.M. Ayres, E.M. Gurol, S.M. Greenberg, J. Rosand, A. Viswanathan, Risk Factors Associated With Early vs Delayed Dementia After Intracerebral Hemorrhage, JAMA neurology 73(8) (2016) 969-76.

[7] A. Tveiten, U. Ljostad, A. Mygland, H. Naess, Functioning of long-term survivors of first-ever intracerebral hemorrhage, Acta Neurol. Scand. 129(4) (2014) 269-75.

[8] L. Xiong, S. Davidsdottir, Y.D. Reijmer, A. Shoamanesh, D. Roongpiboonsopit, S.

Thanprasertsuk, S. Martinez-Ramirez, A. Charidimou, A.M. Ayres, P. Fotiadis, E. Gurol, D.L. Blacker, S.M. Greenberg, A. Viswanathan, Cognitive Profile and its Association with Neuroimaging Markers of Non-Demented Cerebral Amyloid Angiopathy Patients in a Stroke Unit, J. Alzheimers Dis. 52(1) (2016) 171-8.

[9] N.F. Case, A. Charlton, A. Zwiers, S. Batool, C.R. McCreary, D.B. Hogan, Z. Ismail, C. Zerna, S.B. Coutts, R. Frayne, B. Goodyear, A. Haffenden, E.E. Smith, Cerebral Amyloid Angiopathy Is 
Associated With Executive Dysfunction and Mild Cognitive Impairment, Stroke; a journal of cerebral circulation 47(8) (2016) 2010-6.

[10] M. Roussel, O. Martinaud, H. Henon, M. Vercelletto, C. Bindschadler, P.A. Joseph, P. Robert, P. Labauge, O. Godefroy, G.S. Group, The Behavioral and Cognitive Executive Disorders of Stroke: The GREFEX Study, PloS one 11(1) (2016) e0147602.

[11] M.J. van Zandvoort, R.P. Kessels, G.M. Nys, E.H. de Haan, L.J. Kappelle, Early neuropsychological evaluation in patients with ischaemic stroke provides valid information, Clin. Neurol. Neurosurg. 107(5) (2005) 385-92.

[12] D.J. Werring, D.W. Frazer, L.J. Coward, N.A. Losseff, H. Watt, L. Cipolotti, M.M. Brown, H.R. Jager, Cognitive dysfunction in patients with cerebral microbleeds on $\mathrm{T} 2 *$-weighted gradient-echo MRI, Brain : a journal of neurology 127(Pt 10) (2004) 2265-75.

[13] K.A. Knudsen, J. Rosand, D. Karluk, S.M. Greenberg, Clinical diagnosis of cerebral amyloid angiopathy: validation of the Boston criteria, Neurology 56(4) (2001) 537-9.

[14] J.M. Wardlaw, E.E. Smith, G.J. Biessels, C. Cordonnier, F. Fazekas, R. Frayne, R.I. Lindley, J.T. O'Brien, F. Barkhof, O.R. Benavente, S.E. Black, C. Brayne, M. Breteler, H. Chabriat, C. Decarli, F.E. de Leeuw, F. Doubal, M. Duering, N.C. Fox, S. Greenberg, V. Hachinski, I. Kilimann, V. Mok, R. Oostenbrugge, L. Pantoni, O. Speck, B.C. Stephan, S. Teipel, A. Viswanathan, D. Werring, C. Chen, C. Smith, M. van Buchem, B. Norrving, P.B. Gorelick, M. Dichgans, S.T.f.R.V.c.o. nEuroimaging, Neuroimaging standards for research into small vessel disease and its contribution to ageing and neurodegeneration, The Lancet. Neurology 12(8) (2013) 822-38.

[15] G.M. Nys, M.J. van Zandvoort, P.L. de Kort, B.P. Jansen, E.H. de Haan, L.J. Kappelle, Cognitive disorders in acute stroke: prevalence and clinical determinants, Cerebrovascular diseases 23(5-6) (2007) 408-16.

[16] H.J. Kim, K. Im, H. Kwon, J.M. Lee, C. Kim, Y.J. Kim, N.Y. Jung, H. Cho, B.S. Ye, Y. Noh, G.H. Kim, E.D. Ko, J.S. Kim, Y.S. Choe, K.H. Lee, S.T. Kim, J.H. Lee, M. Ewers, M.W. Weiner, D.L. Na, S.W. Seo, Clinical effect of white matter network disruption related to amyloid and small vessel disease, Neurology (2015). 
[17] H.J. Kim, K. Im, H. Kwon, J.M. Lee, B.S. Ye, Y.J. Kim, H. Cho, Y.S. Choe, K.H. Lee, S.T. Kim, J.S. Kim, J.H. Lee, D.L. Na, S.W. Seo, Effects of amyloid and small vessel disease on white matter network disruption, J. Alzheimers Dis. 44(3) (2015) 963-75.

[18] A.K. Dey, V. Stamenova, G. Turner, S.E. Black, B. Levine, Pathoconnectomics of cognitive impairment in small vessel disease: A systematic review, Alzheimer's \& dementia : the journal of the Alzheimer's Association 12(7) (2016) 831-45.

[19] J. Duncan, The structure of cognition: attentional episodes in mind and brain, Neuron 80(1) (2013) 35-50. 